\title{
Update on mitochondrial fatty acid oxidation disorders
}

\author{
Ute Spiekerkoetter • Ertan Mayatepek
}

Published online: 15 September 2010

(C) SSIEM and Springer 2010

Inherited deficiencies of mitochondrial fatty acid oxidation (FAO) are of special importance for metabolic physicians as they are nowadays targets of newborn screening in many countries, and many can be efficiently treated with relatively simple interventions or preventive measures. There has been tremendous progress in our understanding of this group of disorders, not at least in the last decade, due to expansion of newborn screening. Nevertheless, there are still many unanswered questions as well as diagnostic and therapeutic challenges. To summarize current knowledge and identify gaps in our understanding, an international symposium was organized in Fulda, Germany, in October 2008. Important conclusions from this symposium are presented in a number of articles collected in this issue of the JIMD.

Mitochondrial FAO plays a pivotal role in energy homeostasis, especially during periods of increased energy demand, competes with glucose as the primary oxidative substrate and is operated at the hormonal, transcriptional, and biochemical level (Houten and Wanders, doi:10.1007/s10545-010-9061-2; Bennett, doi:10.1007/s10545-010-9170-y). FAO disorders may be caused by mutations in more than 10 genes coding for the various enzymes and transporters involved in the pathway. As a group, FAO disorders belong to the most prevalent monogenic conditions worldwide; figures derived from newborn screening in Australia, Germany, and the USA give a combined incidence of approximately 1:9,300 (Lindner et al., doi:10.1007/s10545-010-9076-8). The identification of FAO

U. Spiekerkoetter $(\bowtie) \cdot$ E. Mayatepek

Department of General Pediatrics, University Children's Hospital,

Moorenstr. 5,

40225 Duesseldorf, Germany

e-mail: ute.spiekerkoetter@uni-duesseldorf.de disorders has been revolutionized through the introduction of acylcarnitine analysis by tandem mass spectrometry; nevertheless, there remains a need to unify confirmatory diagnostic procedures, e.g., through specific enzyme analyses as outlined by Wanders et al. (doi:10.1007/s10545-010-9104-8). Mutation analyses may not only confirm the diagnosis but may also help to explain differences in the phenotypic presentation of patients with the same enzyme deficiency. Different mutations have different effects on protein function, and some missense mutations result in misfolded but stable proteins that have other effects on the integrity and function of the putative FAO metabolon than pure null mutations that cause absence of the protein (Gregerson and Olson, doi:10.1007/s10545-010-9046-1). Systematic clinical and experimental studies are required to further elucidate the pathophysiology of the different FAO disorders; examination of mouse models, which resemble the human conditions, have already produced promising results (Spiekerkoetter and Wood, doi:10.1007/s10545-010-9121-7).

For long-chain fatty acid oxidation defects and medium chain acyl CoA dehydrogenase (MCAD) deficiency, implementation of newborn screening has significantly reduced morbidity and mortality but prediction of the expected clinical phenotype is difficult (Spiekerkoetter, doi:10.1007/s10545-010-9090-x; Wilcken, doi:10.1007/ s10545-009-9001-1). SCAD deficiency is a notable example of how difficult it can be to distinguish "disease", "susceptibility/risk factor", or "non-disease", particularly with regard to the common variants that are associated with considerable residual activity (van Maldegem et al., doi:10.1007/s10545-010-9080-z). The identification of attenuated forms such as "mild MCAD deficiency" has caused concern as some children may be diagnosed with a "disease" even though clinical problems may only arise under extreme circumstances or not at all. On the other hand, successful prevention of metabolic decompensation 
in children with severe MCAD deficiency by avoidance of fasting must not lead to a neglect of the continuing risks in adulthood, and efforts need to be made to properly inform and educate affected adolescents and adults (Schatz and Ensenauer, doi:10.1007/s10545-0109115-5). Evidence-based recommendations for optimal monitoring and treatment in patients with FAO disorders are only starting to emerge; they will need to take into consideration both universal guidelines as well as individual tailoring (Lund et al., doi:10.1007/s10545-009-9000-2; Spiekerkoetter et al., doi:10.1007/s10545-010-9188-1). We hope that the collection of articles on FAO disorders in this issue of the JIMD will stimulate discussion, collaboration, and research, which ultimately will lead to improved diagnosis, management, and outcome, in patients with these disorders. 